\title{
Research Paper \\ Comparing Plasma Levels of C-Reactive Protein, Interleukin-10 and -15 in Physically Active and Sedentary Postmenopausal Women
}

\author{
*Mona Sarhadi ${ }^{1}$, Mokhtar Nasiri Farsani², Kiana Hassanzadeh ${ }^{3}$
}

1. Departments of Physical Education and Sport Sciences, Faculty of Humanities, Takestan Branch, Islamic Azad University, Takestan, Iran.

2. Departments of Physical Education and Sport Sciences, Faculty of Management and Accounting, Qazvin Branch, Islamic Azad University, Qazvin, Iran

3. Department of Sport Physiology, Faculty of Physical Education and Sport Sciences, Karaj Branch, Islamic Azad University, Karaj, Iran.

Received: 17 Sep. 2016 Accepted: 22 Dec. 2016

Key words: Regular physical activity, Postmenopausal women, C-reactive protein, Interleukin-15; Interleukin-10
Chtation: Sarhadi M, Nasiri Farsani M, Hassanzadeh K. [Comparing Plasma Levels of C-Reactive Protein, Interleukin-10 and -15 in Physically Active and Sedentary Postmenopausal Women (Persian)]. Iranian Journal of Ageing; 2017; 12(1):104-115. http://dx.doi. org/10.21859/sija-1201104

http://dx.doi.org/10.21859/sija-1201104

\begin{abstract}
A B STRACT
Objectives Lower levels of inflammatory cytokines and higher levels of anti-inflammatory cytokines are associated with increased life expectancy. However, the effects of regular physical activity on these cytokines in postmenopausal women have not been investigated very well.

Methods \& Materials Fifteen active and 15 inactive postmenopausal women (aged between 50-60 years) were recruited randomly. The exclusion criteria included chronic diseases, smoking, and surgeries in the past year. Also, more than a year should have passed since all subjects experienced natural menopause. Active postmenopausal women should have regular physical activity included walking, jogging, and resistance exercises and they should have performed these exercises three times a week within the past 6-12 months. After 12 hours of fasting, blood samples were taken in rest. Blood sample was centrifuged at $4000 \mathrm{rpm}$ for 5 minutes, and the serum was frozen and stored at $-80^{\circ} \mathrm{C}$ until biochemical analyses were performed. Independent $t$ test was used to compare quantitative variables, and $\alpha$ level for statistical significance was set at $\mathrm{P} \leq 0.05$.

Results Regular physical activity brings about a significant decrease in C-reactive protein, significant increase in IL-15 and non-significant increase in IL-10 in active postmenopausal women in comparison to inactive postmenopausal women.

Conclusion Regular physical activity may result in lower incidence of chronic diseases in postmenopausal women via decreased inflammatory cytokines and increased anti-inflammatory cytokines and could, therefore, play a role in higher life expectancy.
\end{abstract}

\section{Extended Abstract}

\section{Objectives}

hronic diseases, including cardiovascular diseases, cancer, lung diseases, and diabetes mellitus are the leading causes of death in the world. Research has shown the relationship between inflammation and chronic diseases [1].Aging leads to slight chronic inflammation, and the proinflammatory cytokine levels of Interleukin-6 (IL-6), tumor necrosis factor- $\alpha$ and C-reactive protein (CRP) among the elderly are increased by 2-4 times compared with young people [2]. Evidence indicates that increased inflammatory cytokines associated with aging is higher in postmeno-

\section{* Corresponding Author:}

Mona Sarhadi, PhD

Address: Department of Physical Education and Sport Sciences, Faculty of Humanities, Takestan Branch, Islamic Azad University, Takestan, Iran. Tel: +98 (912) 7685080

E-mail: monasarhadi@yahoo.com 
pausal women [3]. Cytokines of IL-10 and IL-15 may contribute in reducing the risk of disease and increasing longevity among the elderly. Given that little or contradictory research has been carried on the effect of regular exercise on cytokines, the present study aimed to study the resting cytokine levels of CRP, IL-10 and IL-15 in physically active postmenopausal women and compare it with sedentary postmenopausal women.

\section{Methods \& Materials}

This study was approved by the Ethics Committee of Qazvin Islamic Azad University. In order to select physically active postmenopausal women, the sports clubs and gyms in Karaj were visited, and notifications and questionnaires were distributed for the purpose of cooperation in research. About 89 questionnaires were completed; of them, 22 subjects were in the age range of 50-60 years and were thus excluded from the study. Some notifications were distributed in the gathering places of old women and public places in order to select sedentary postmenopausal women. Finally, 52 people volunteered out of which 17 were eligible. The exclusion criteria were having chronic diseases, smoking, and surgeries in the past year. Also, more than a year should have passed since all subjects experienced natural menopause. Active postmenopausal women should have regular physical activity, including walking, jogging, and resistance exercises and they should also have performed these exercises three times a week within the past 6-12 months.

Finally, 15 active and 15 sedentary postmenopausal women were selected out of the qualified individuals through simple random sampling method. The exercise performed by the active group was a combination of hiking, jogging, and strength exercises.The subjects participated in the briefing one week before the study, and their height, weight, body mass index, and body fat percentages were measured. The subjects were asked to refrain from strenuous physical activity 48 hours before blood sampling and take dinner before 9 PM before measurement. On the measurement day, after
12 hours of fasting, blood samples were taken from left-hand vein in sitting and resting positions. Serum was separated from the blood by a centrifuge at 4000 rpm for 5 minutes and then used to measure IL-10, IL15 , and CRP. Independent $t$ test was used to compare the results between two groups. All statistical analyses were performed at a significance level of 0.05 .

\section{Results}

The Kolmogorov-Smirnov test results showed that all data variables were normally distributed. Also, according to Levene's test results, the variance of the groups was homogeneous. Thus, there was the presumption of using parametric tests. The average age was 54.20 years in the active group and 54.06 years in the sedentary group while the mean height was $163.43 \mathrm{~cm}$ in the active group and $161.36 \mathrm{~cm}$ in the sedentary group. The average weight was $63.41 \mathrm{~kg}$ in the active group and $65.20 \mathrm{~kg}$ in the sedentary group. The mean body mass index was $23.72 \mathrm{~kg} / \mathrm{m}^{2}$ in the active group and $25.02 \mathrm{~kg} / \mathrm{m}^{2}$ in the sedentary group, and finally the average percentage of body fat was $20.86 \%$ in the active group and $23.06 \%$ in the sedentary group. Independent t test was used to study the homogeneity of the groups in terms of anthropometric indices, and the results showed that the groups were homogeneously divided for the index of age $(\mathrm{P}=0.896)$, weight $(\mathrm{P}=0.371)$, height $(\mathrm{P}=0.154)$ and body fat percentage $(\mathrm{P}=0.153)$. There was a significant difference between the groups with regard to the index of $\mathrm{BMI}(\mathrm{P}=0.046)$.

Table 1 shows independent $t$ test results on fasting plasma levels of CRP, IL-10, and IL-15 in physically active and sedentary women. As seen in the Table, fasting plasma levels of the cytokine IL-10 are higher in active postmenopausal women but not statistically significant $(\mathrm{P}=0.228)$. Therefore, regular exercise insignificantly increased the cytokines IL-10 in active postmenopausal women.

Also, as shown in Table 1, fasting plasma levels of the cytokine IL-15 are higher in active postmenopausal

Table 1. Intergroup comparisons of CRP, IL-10 and IL-15 values (mean \pm SD) in active and sedentary postmenopausal women

\begin{tabular}{|c|c|c|c|c|c|}
\hline Variable & Active Postmenopausal Women & Sedentary Postmenopausal Women & df & $\mathbf{t}$ & Significance \\
\hline $\mathrm{CRP}(\mathrm{mg} / \mathrm{l})$ & $51.02 \pm 2.1$ & $25.86 \pm 3.0$ & 28 & 2.147 & 0.041 \\
\hline IL-10 (pg/ml) & $72.41 \pm 1.0$ & $51.52 \pm 10$ & 28 & 1.233 & 0.228 \\
\hline $\mathrm{IL}-15$ (pg/ml) & $70.52 \pm 3.0$ & $60.64 \pm 2.0$ & 28 & 2.213 & 0.035 \\
\hline
\end{tabular}


women $(\mathrm{P}=0.035)$. This indicated that regular exercise significantly increased the cytokine IL-15 in active postmenopausal women. As can be seen in Table 1, fasting plasma levels of CRP are significantly lower in active postmenopausal women $(\mathrm{P}=0.041)$. So, regular exercise significantly decreased CRP in active postmenopausal women. The results suggest that regular exercise leads to a significant decrease in CRP, significant increase in IL-15 and insignificant increase in IL10 among active postmenopausal women compared to sedentary postmenopausal women.

\section{Conclusion}

Regular exercise fights against chronic diseases, in all probability, by reducing inflammation and increasing anti-inflammatory cytokines in postmenopausal women and may thereby increase the effective life expectancy. Studies taking into account large populations and people with an average age of over 60 years have suggested an inverse association between physical activity and inflammatory biomarkers $[4,5]$. The effects of physical exercise on systemic inflammation occur through weight loss and adipose tissue loss [6], and it is likely that the difference between groups is due to the higher body mass index and body fat percentage in the sedentary group. Changes in immunological parameters because of exercises depend on age, sex, physical fitness, duration, intensity, and the type of activity [7]. Cytokine secretion mechanisms in relation to exercise are very complex and poorly understood. Interpretation of results in this area requires further studies.

\section{Acknowledgments}

This research did not receive any specific grant from funding agencies in the public, commercial, or not-forprofit sectors.

\section{Conflict of Interest}

The authors declared no conflicts of interest. 


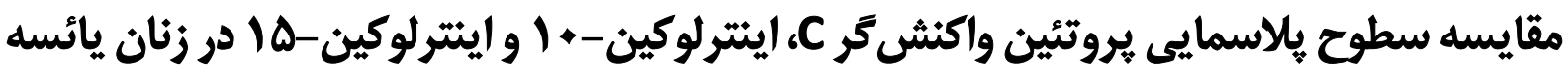

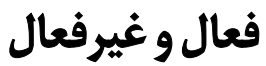

\author{
"مونا سرحدى'، مختار نصيرى فارسانى'، كيانا حسنزاده" \\ ا - كروه تربيت بدنى و علوم ورزشى، دانشكده علوم انسانى، واحد ثاكستان، دانشكاه آزاد اسلامى، تاكستان، ايران.

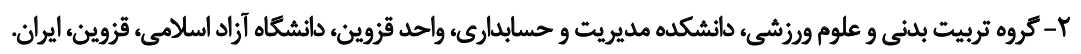

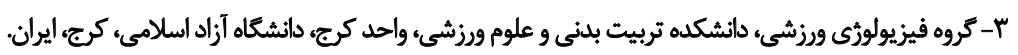

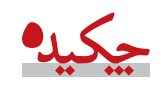

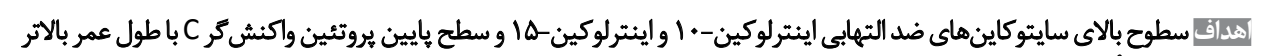

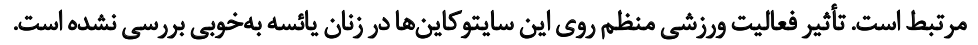

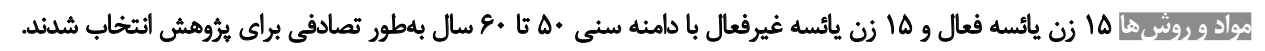

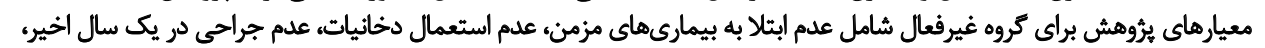

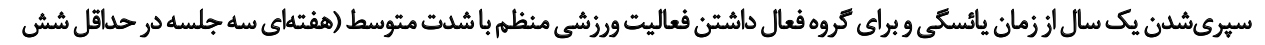

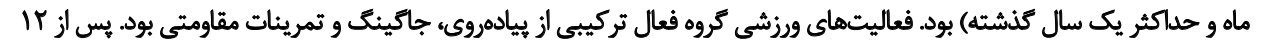

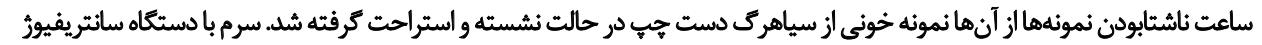

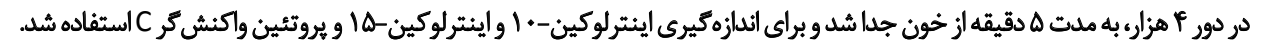

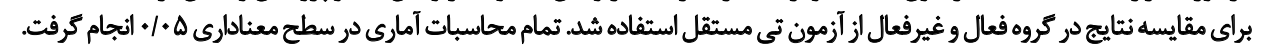

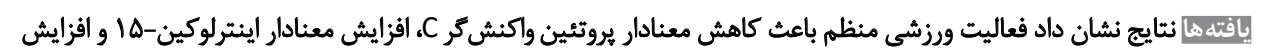

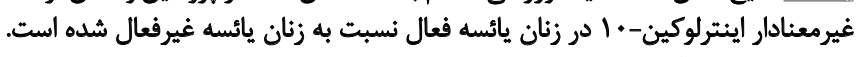

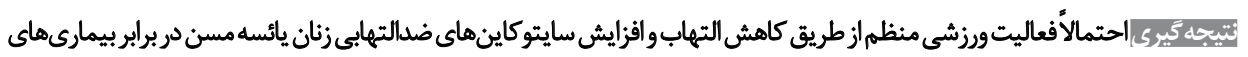
مزمن مقابله مي كند و شايد از اين طريق در افزايش طول عمر مؤثر باشئ.
\end{abstract}

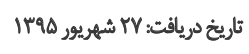

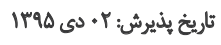

$$
\begin{aligned}
& \text { كليدوارٔهها: } \\
& \text { زعاليت ورزشى منظه، } \\
& \text { زنان يائسه، يروتثين منين } \\
& \text { واكنش كر C، اينترلوكين }
\end{aligned}
$$

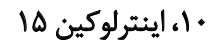

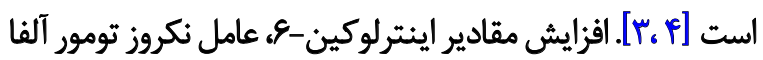

datêo

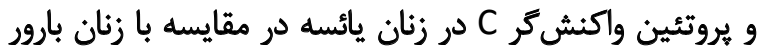

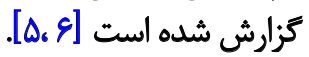

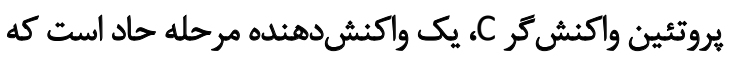

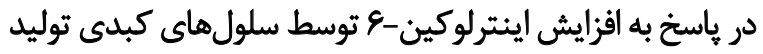

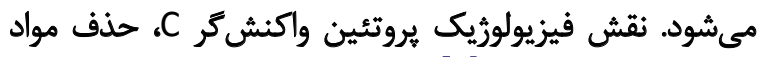

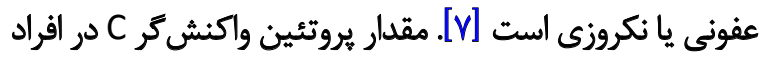

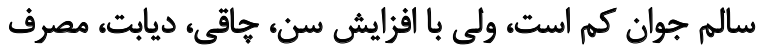

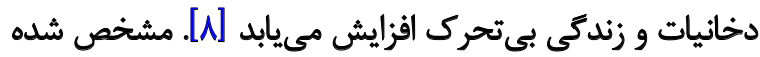

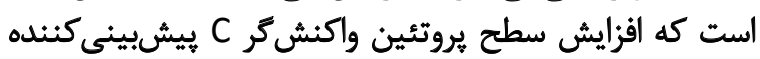

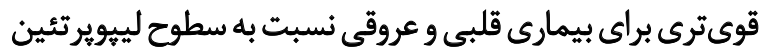

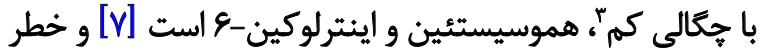

بيمارىهاى مزمن عامل اصلى مرگتومير در جهان هستئد

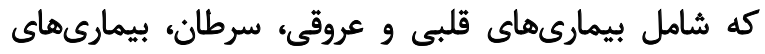

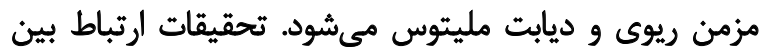

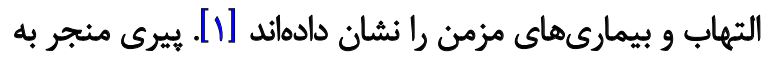

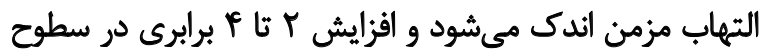

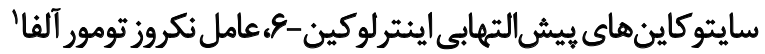

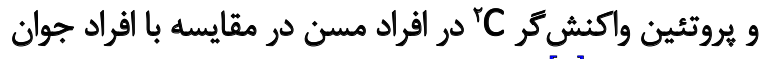

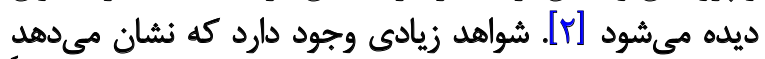

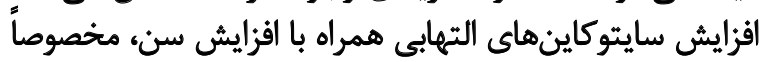
بعد از يائسكى در زنان به دليل كاهش التهابي عملكرد تخمدانى افئ بالاتر

1. Tumor Necrosis Factor alpha (TNF $\alpha$ )

2. C-Reactive Protein (CRP) -

$$
\begin{aligned}
& \text { * نويسئده مسئول: } \\
& \text { دكتر مونا سرحدى مدئ } \\
& \text { نشانى، : تاكستان، واحد تاكستان، داتشعاه آزاد اسلامى، دانشكده علوم انسانى، تروه تربيت بدنى و علوم ورزشى. }
\end{aligned}
$$

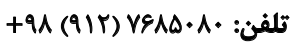

$$
\begin{aligned}
& \text { بست الكترونيكى: monasarhadi@yahoo.com }
\end{aligned}
$$


غلظت سرمى اينتر لوكين-10 در افرادى كه طول عمر غيرمعمول

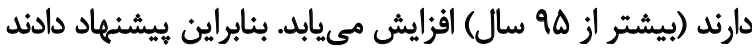

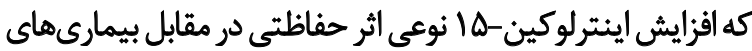

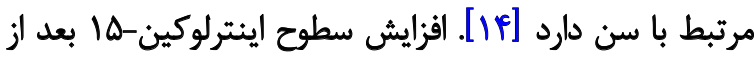

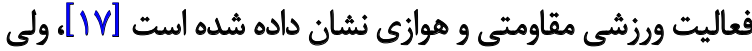

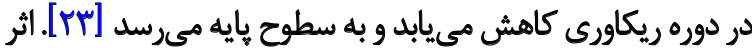

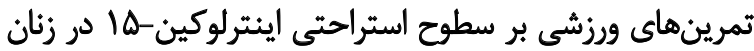

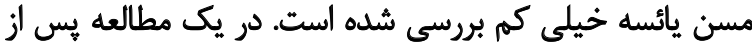

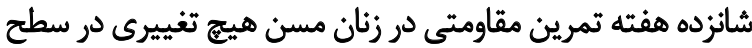

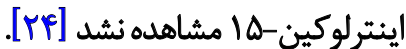

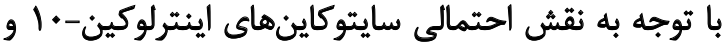

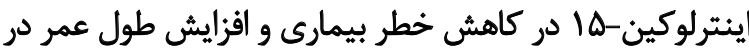

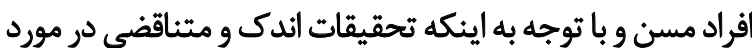

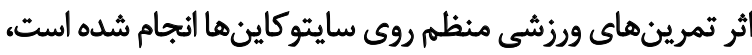
هدف مطالعه حاضر بررسى سطوح استراحتى سينى سايتوكاينهاي

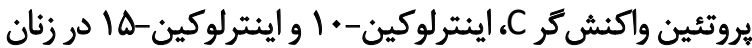

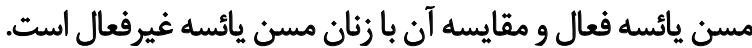

$$
\text { روش مطاله }
$$

براى انتخاب زنان يائسه فعال به باشكاهها و سالنهاى ورزشى

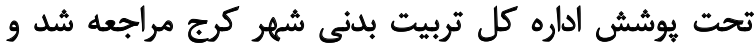

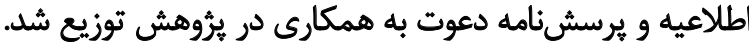

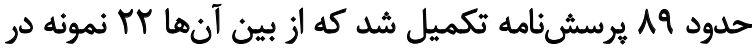

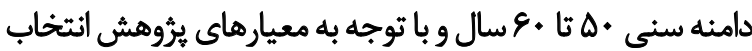

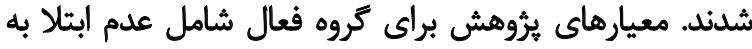

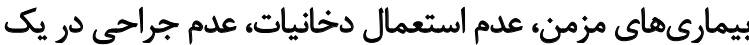

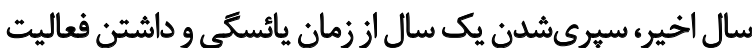

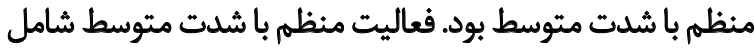

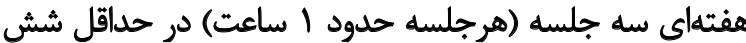

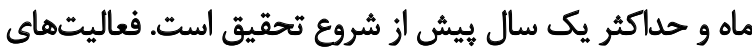

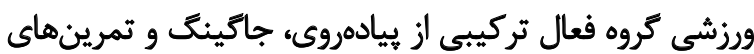

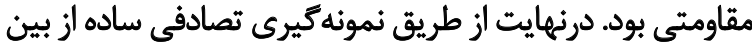

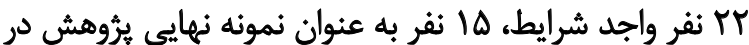

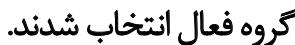

براى انتخاب زنان يائسه غيرفعال با نصب اطلاعيه در محل

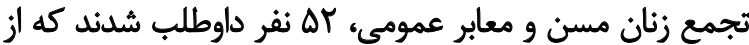

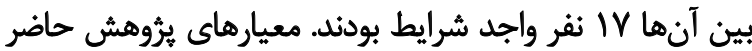

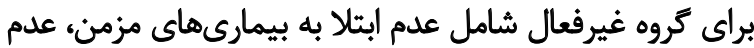

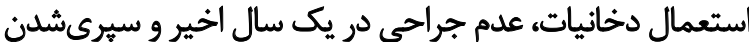

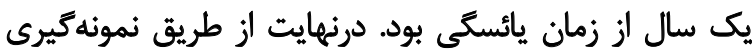

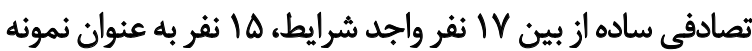
نهايي يرؤهش در تروه غيرفعال انتخاب شدئد. يس از تكميل رضايتنامه و يرسشنامه وضعيت سلامتى،

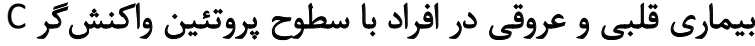

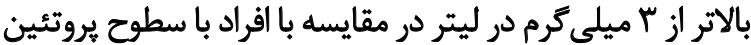

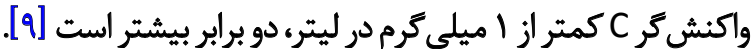
مطالعات روى جوامع بزرى و در افراد با ميانكين سنى بالاي

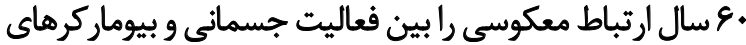

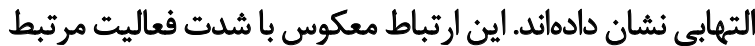

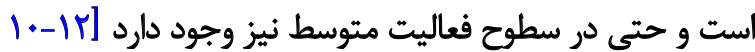

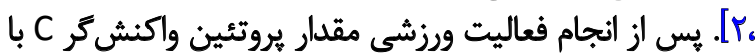

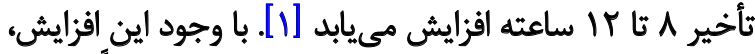

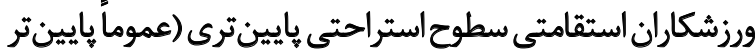

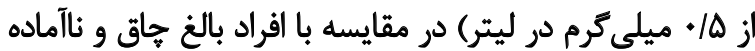

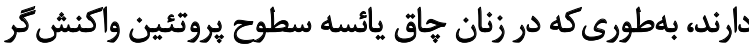

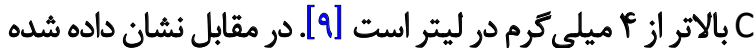

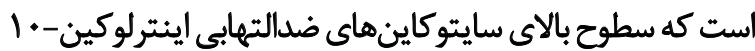

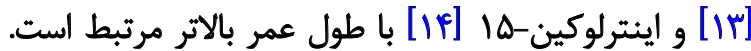

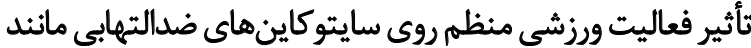

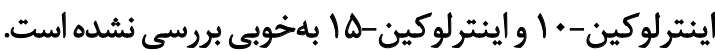

سايتوكاين اينترلوكين- - ا يك سايتوكاين ضدالتهابي است كه

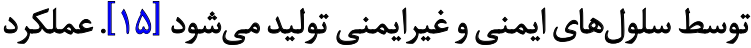

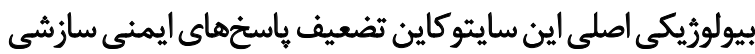

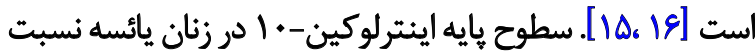

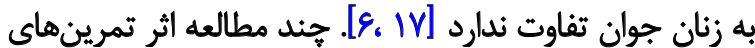

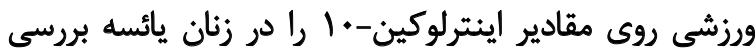

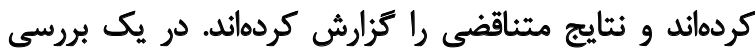

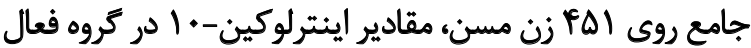

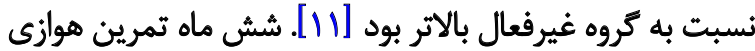

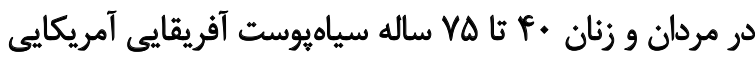

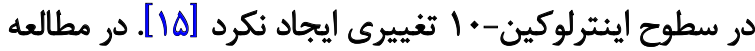

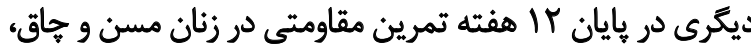

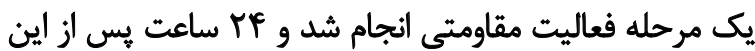

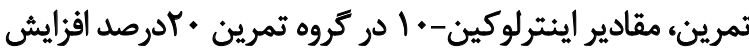

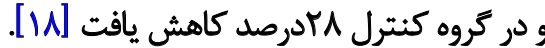

سايتوكاين اينترلوكين-10 به عنوان عامل رشد شناسايى شده

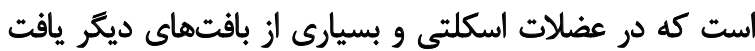

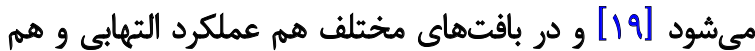

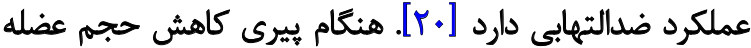

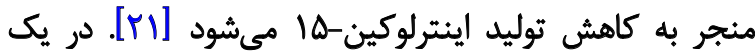

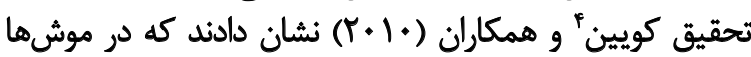

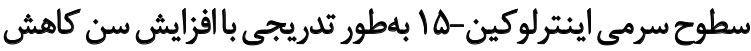

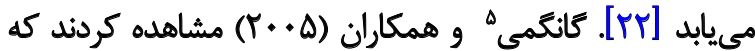

4. Quinn

5. Gangemi 
بدن و درصد جربي بلدن در جدول شماره إ آورده شده است. نثايج

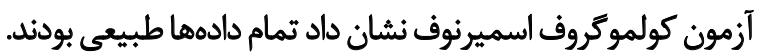

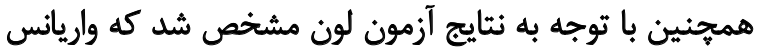

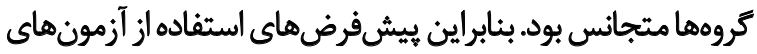

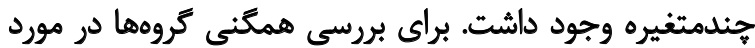

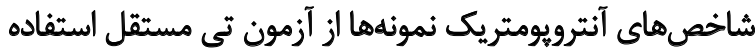

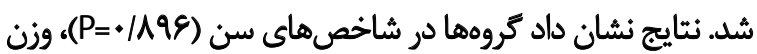

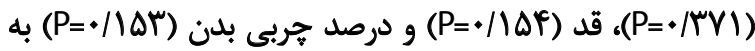

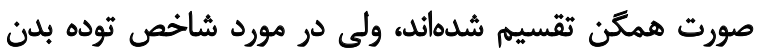
(P=. بين كروهها اختلاف معنادارى وجود دارد.

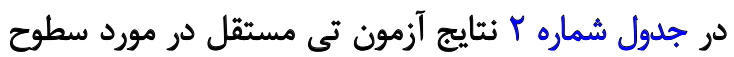

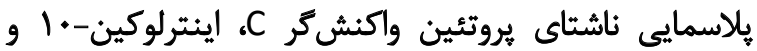

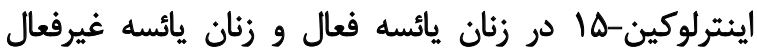

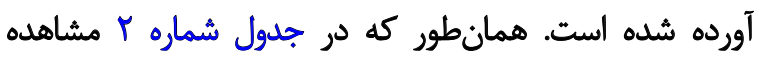

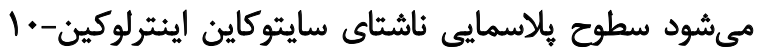

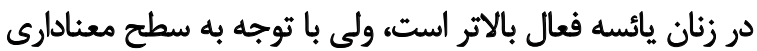

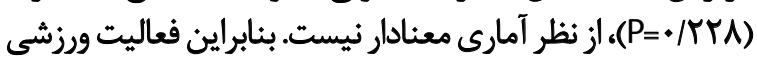

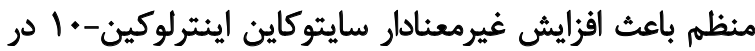
زنان يائسه فعال شده است.

همانطور كه در جدول شماره Y مشاهده مي إشود سطوح

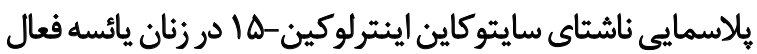

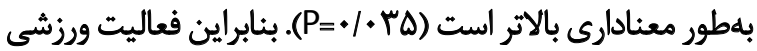

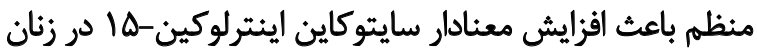

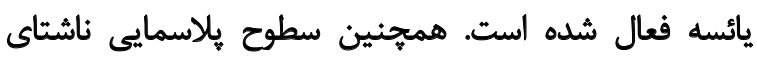

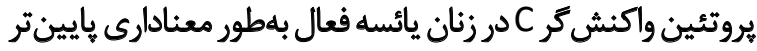

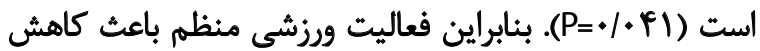

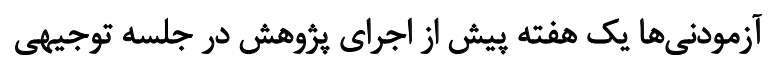

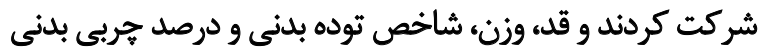

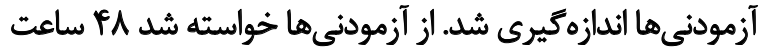

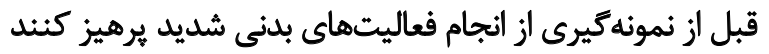

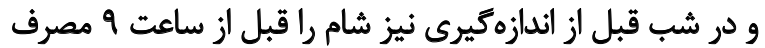

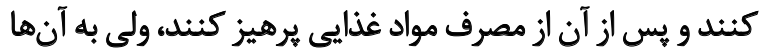

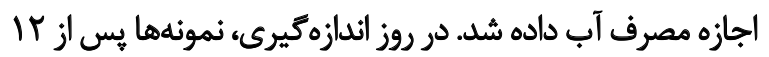

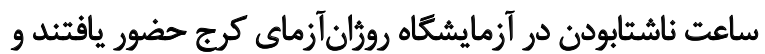

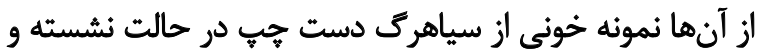
استراحت كرفته شد.

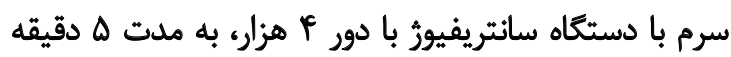

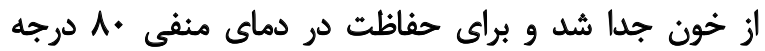

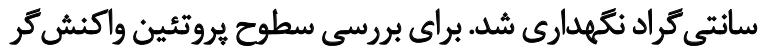

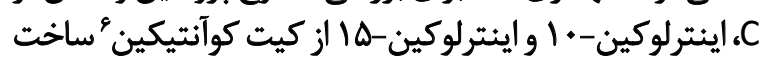

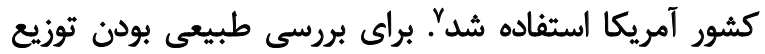

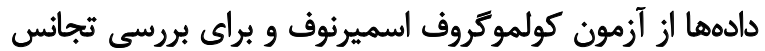

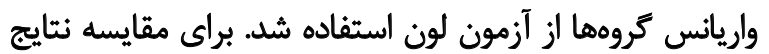

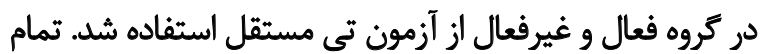

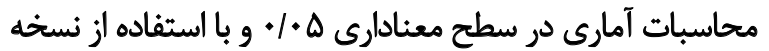

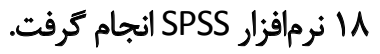

ياقتهاهها

ويرَكىهاى فردى آزمودنى ها شامل سن، قد، وزن، شاخص توده

6. Quantikine

7. Quantikine; R \& D systems, MinneaPolis, MN-USA

جدول ا. ميانكين و انحرافمعيار شاخصهاى آنترويومتريك افراد شركت كنيده در بروهش

\begin{tabular}{|c|c|c|c|c|c|}
\hline درصد هربى & توده بدنى (kg/m²) & وزن (كيلوكرم) & قد (سانتىمتر) & سن (سال) & كروه \\
\hline$r \cdot / r \pm N \& / M$ & $m / \Lambda \pm V / \Lambda$. & $e^{M} / \Delta \pm P I / R$ & $\mid E N / P \pm P T / N E$ & $\Delta Y / Y \pm Y \cdot / M$ & زنان يائسه فعال (n=10) \\
\hline$r r / t_{ \pm+} \varepsilon / r^{2}$ & $r \Delta / / \pm+r / R r$ & $8 \Delta / F \pm r \cdot / q r$ & $|\&| / T \pm K E / \Delta \varphi$ & $\Delta \varphi / Y \pm \cdot F / \& T^{\circ}$ & زنان يائسه غيرفعال (A) \\
\hline
\end{tabular}

故

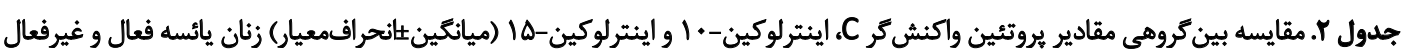

\begin{tabular}{|c|c|c|c|c|c|}
\hline معنادارى & مقدار تى & درجه آزادي & زنان يائسه غير فعال & زنان يائسه فعال & مثئير \\
\hline $.1+41$ & T/IFV & rA & $r / \cdot \pm r \Delta / N \mathcal{A}$ & $r / \lambda \pm \Delta V / \cdot r$ & 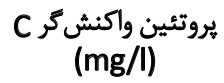 \\
\hline ./TMA & שחז/V & rA & $V / \cdot \pm \Delta V / \Delta r$ & $V / \pm V T / F I$ & ايتترلوكين-1) \\
\hline $1 . r \Delta$ & r/MIT & ra & $Y / \cdot \pm 8 \cdot 194$ & $r / \cdot \pm+V / \Delta T$ & $\begin{array}{c}\text { اينتر لوكين-10 } \\
\text { (pg/ml) }\end{array}$ \\
\hline
\end{tabular}




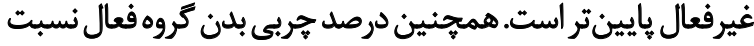

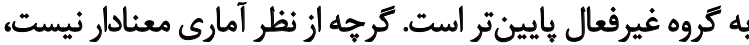

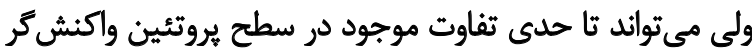

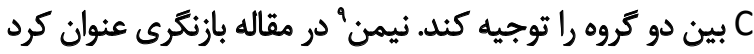

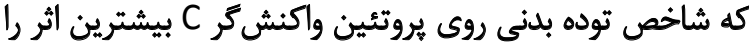

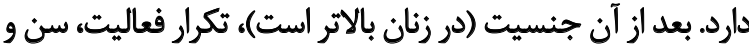

وضعيت مصرف دخانيات بيشترين اثر را دارد [9].

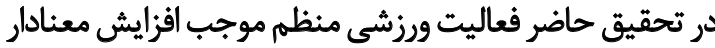

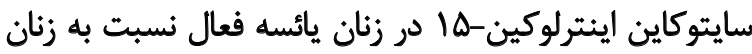

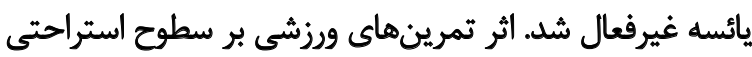

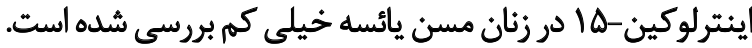

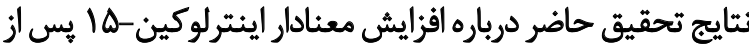

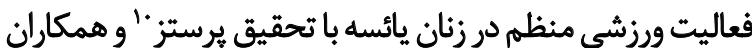

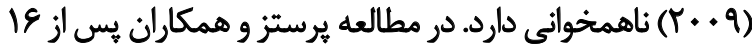

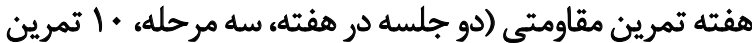

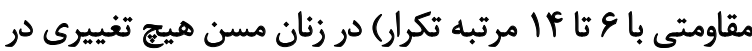

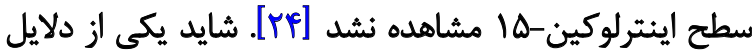

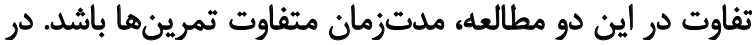

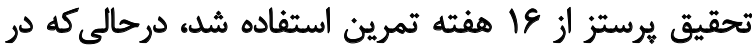

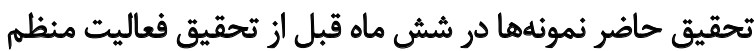

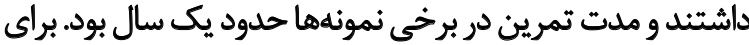

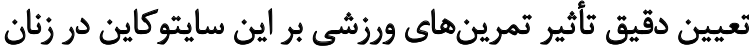
مسن يائسه نياز به مطالعات بيشترى تمين است

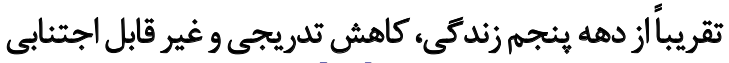

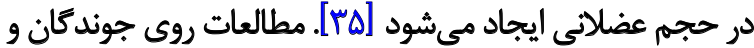

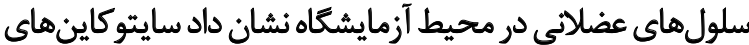

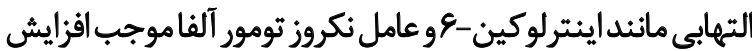

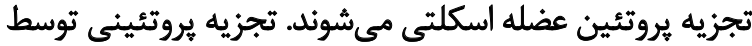

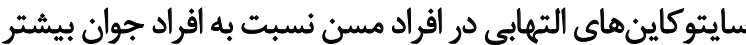

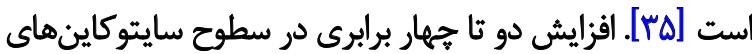

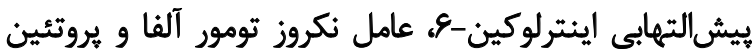

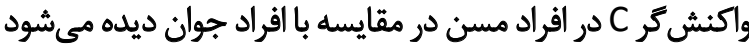

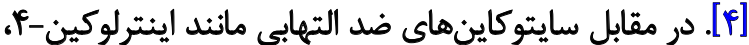

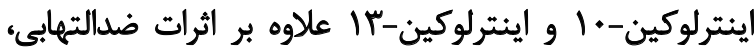

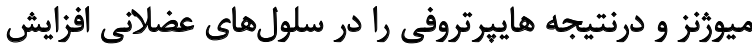

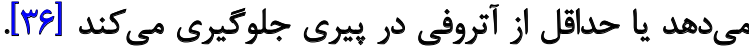

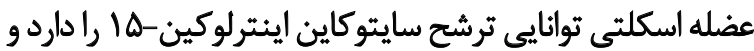

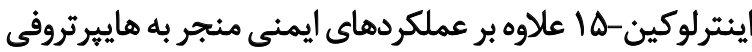

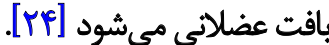
سايتوكاين اينترلوكين-ها اتمايز ميوسيتهاو تارهاى عضلانى

9. Nieman (2012)

10. Prestest
معنادار يروتئين واكنش C C در زنان يائسه فعال شده است.

\section{ثب}

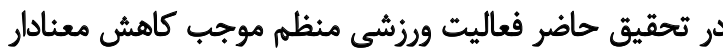

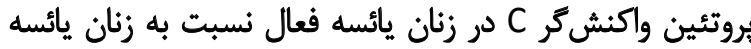

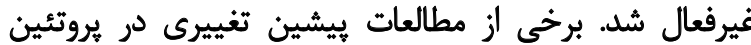

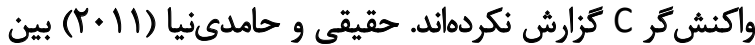

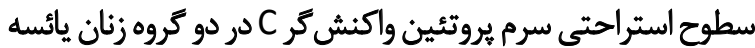

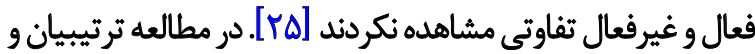

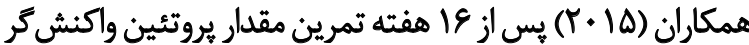

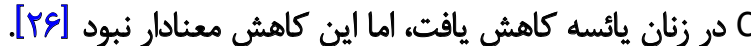

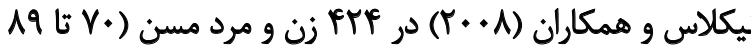

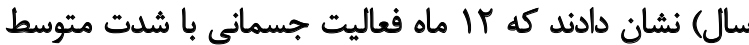

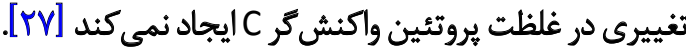

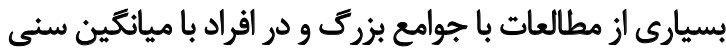

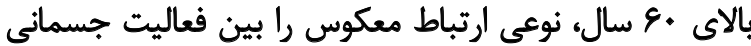

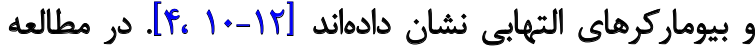

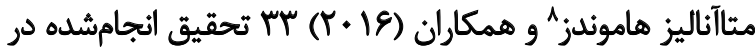

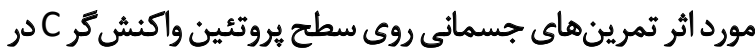

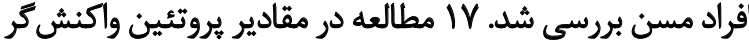

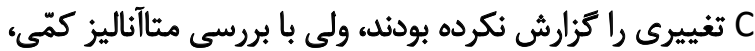

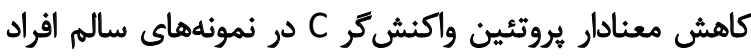

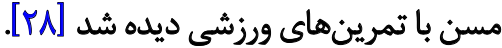
ممكن است كاهش يُروتئين واكنش

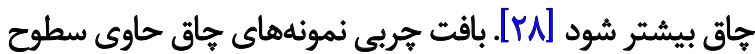

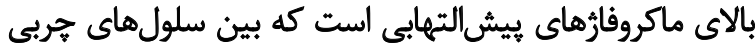

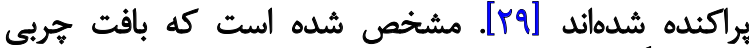

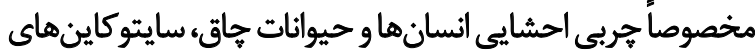

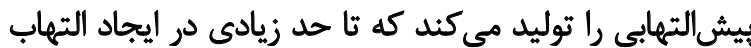

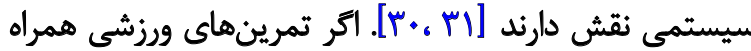

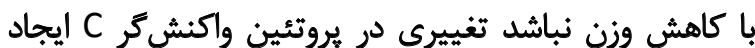

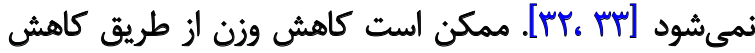

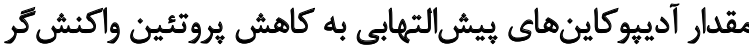

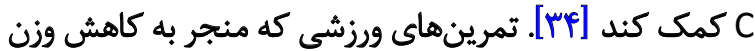

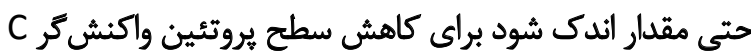

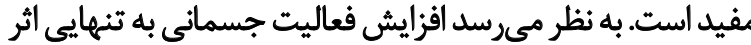

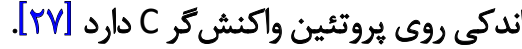

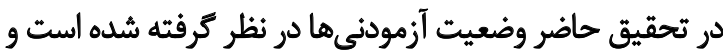

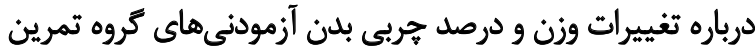

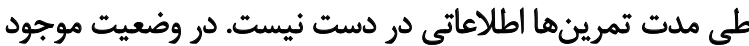

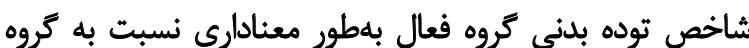

8. Hammonds 


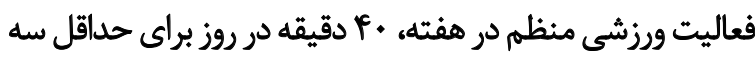

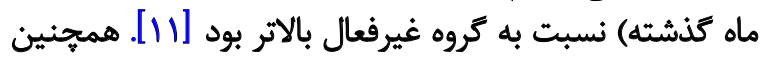

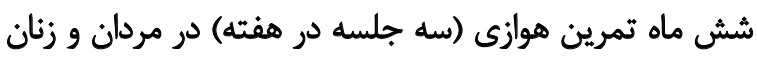

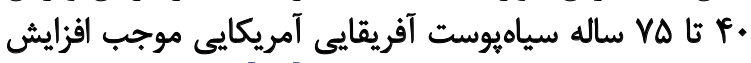

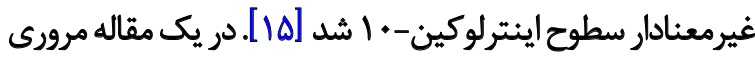

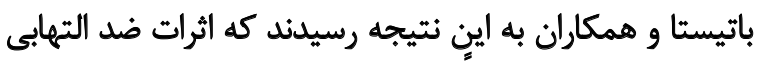

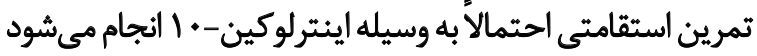

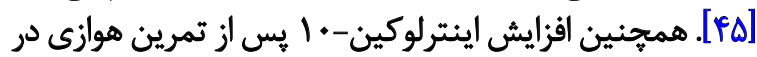

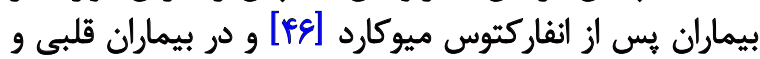
عروقى [FV] نشان داده شد.

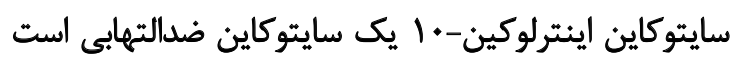

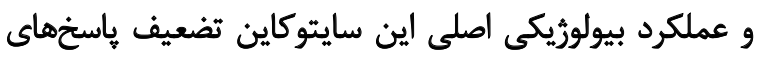

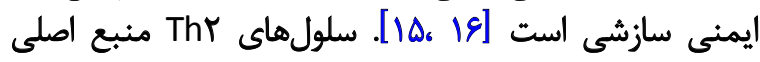

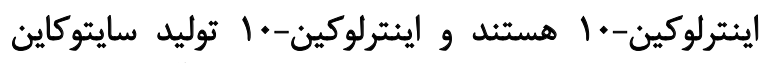

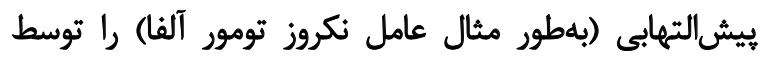

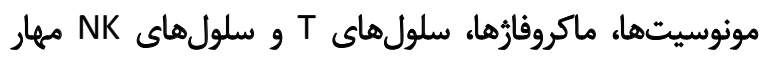

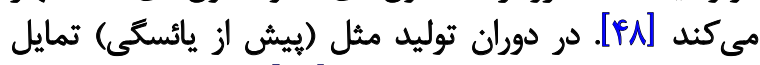

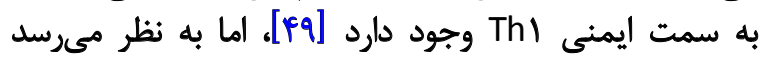

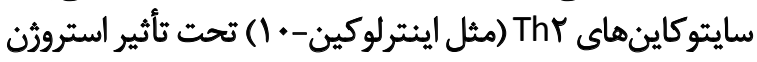

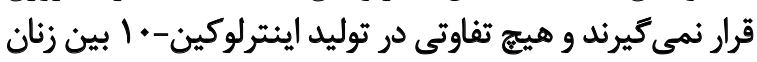

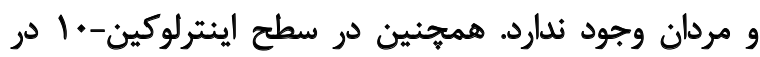

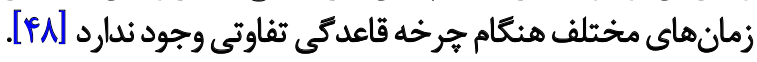

در مورد يائسكى نتايج متفاوتى كزارش شده است. درحالى ماكه

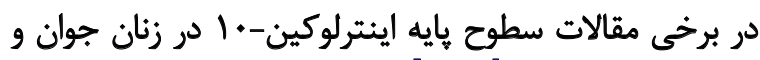

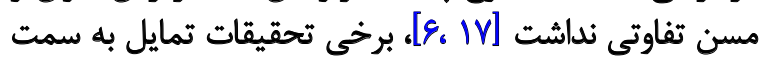

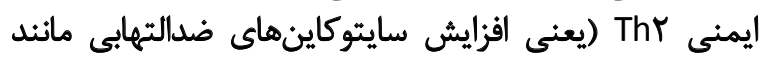

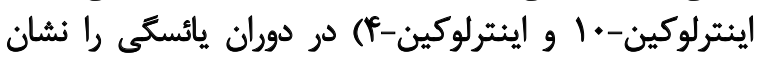

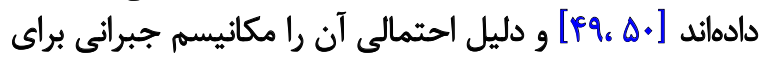

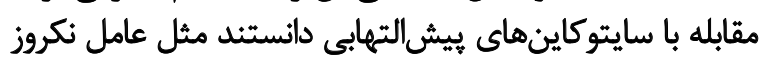

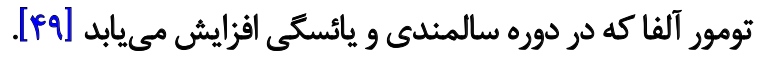

روى مردان مسن نيز تحقيقاتى انجام شده است.

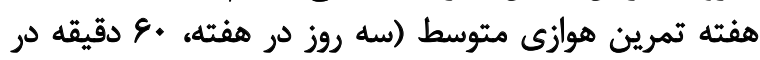

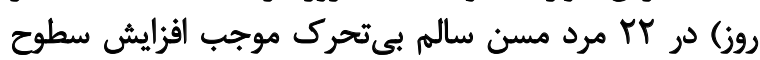

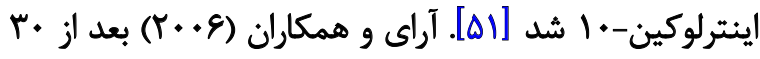

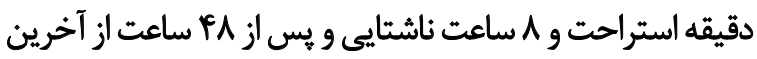

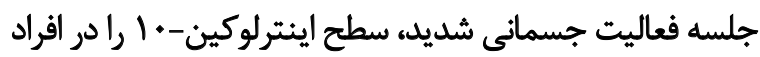

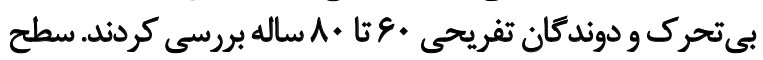

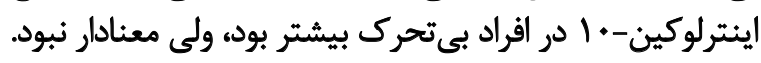

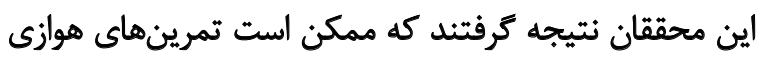

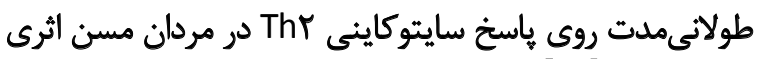

نداشته باشد [هY].

نأثير تمرينهاى جسمانى روى التهاب سيستمى از طريق

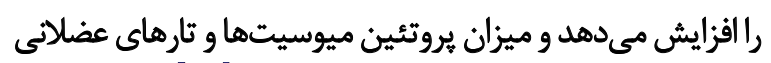

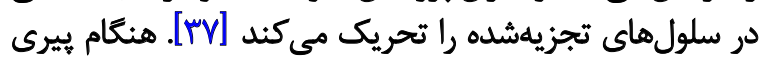

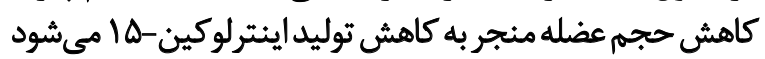

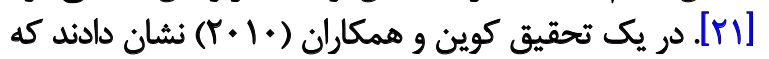

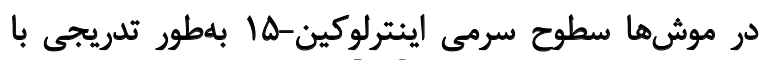
افزايش سن كاهش مى يابد [Tr.

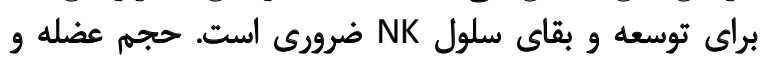

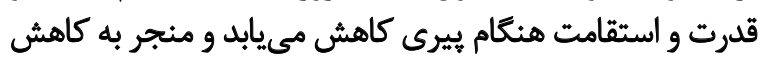

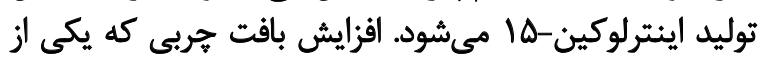

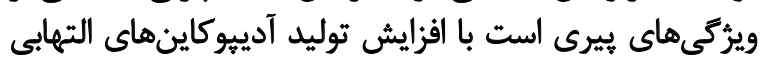

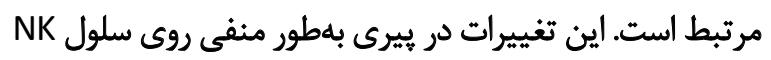

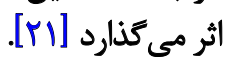

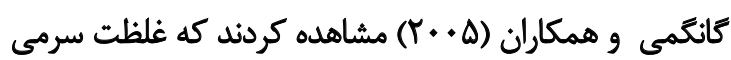

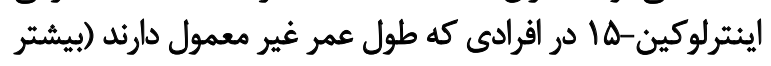

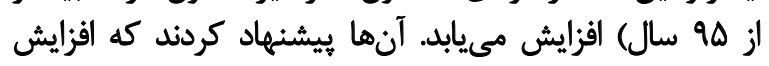

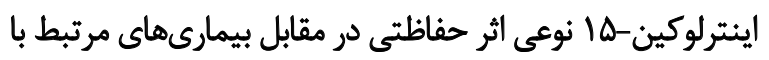

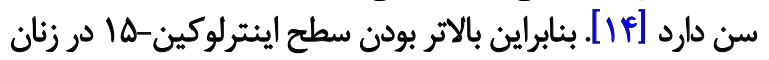

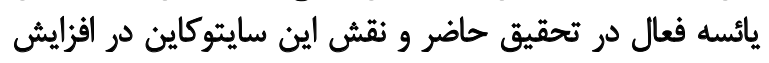

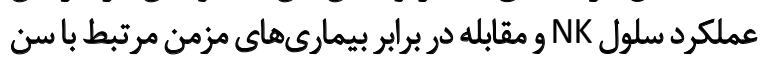

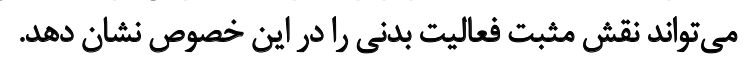

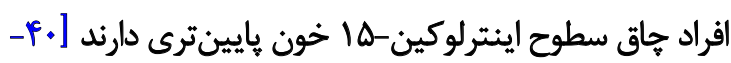

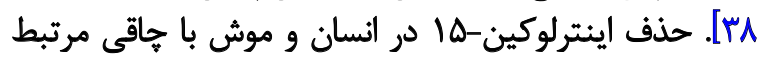

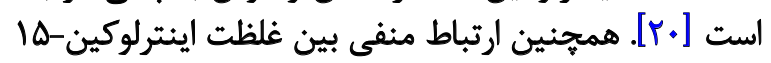

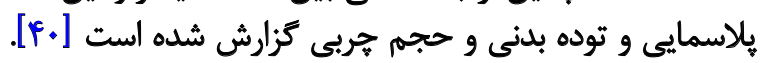

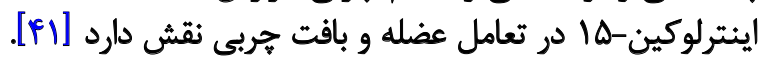

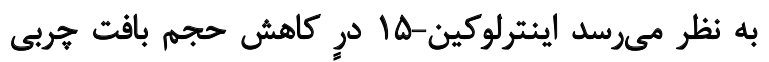

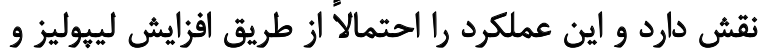

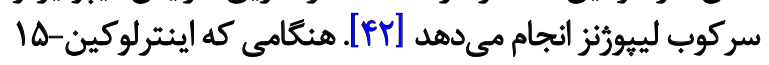

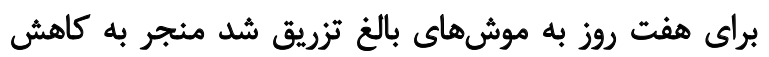

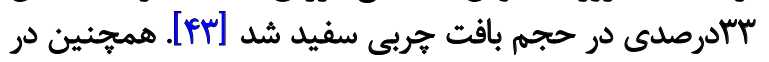

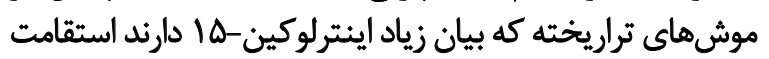

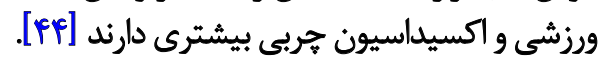

در مطالعه حاضر شاخص توده بدنى زنان يائسه فعال و غيرفعال

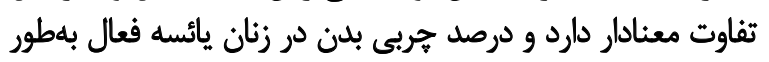

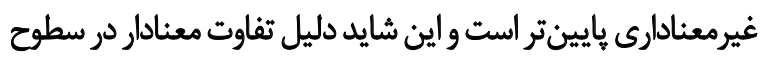

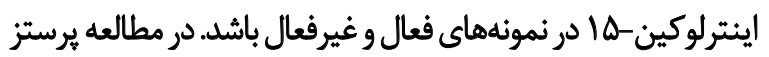

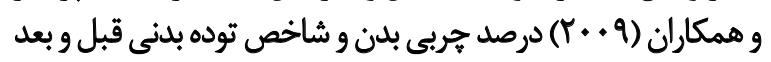

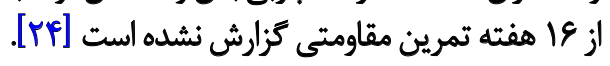

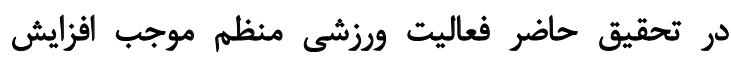

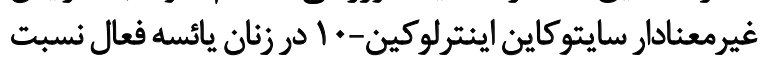

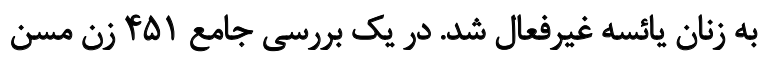

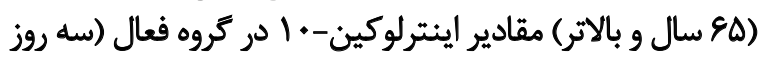


كاهش وزن و كاهش بافت جربى صورت مي كيرد [ـها]. بلهور

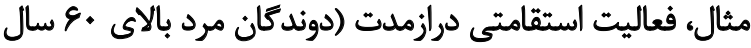

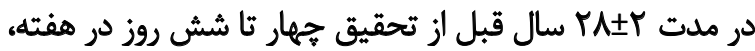

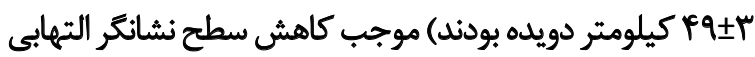

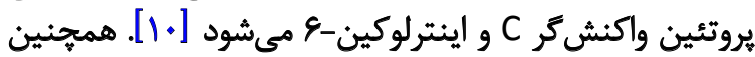

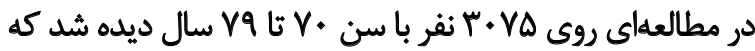

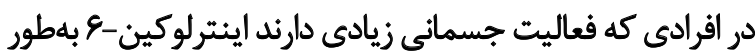

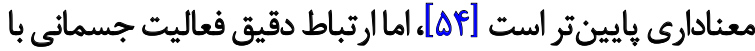

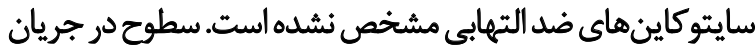

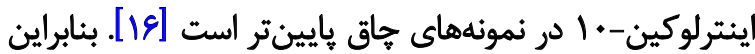

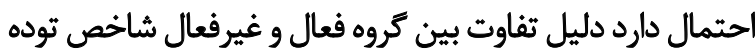

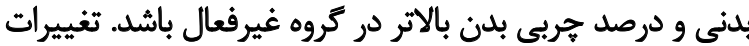

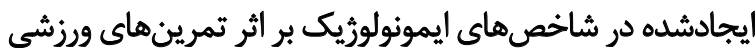

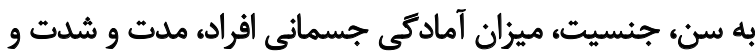

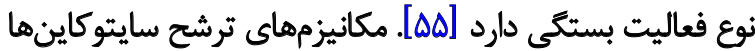

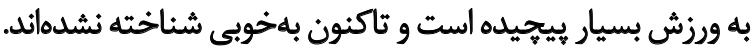

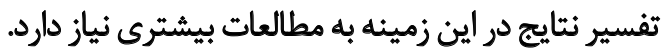

\section{نتيجنكيرى ننهايي}

نتايجتحقيق حاضرنشان دادفعاليت ورزشى منظم باعث كاهش

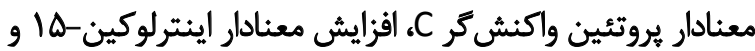

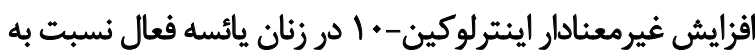

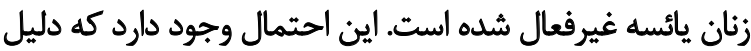

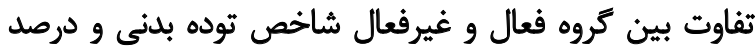

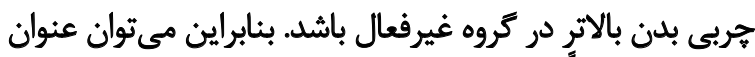

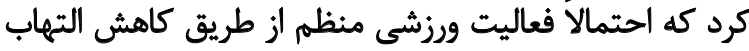

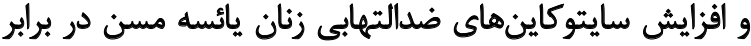

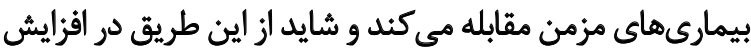

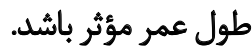
تشكر و قدردانى

از تمامى آزمودنى هاى حاضر در يُروهش و از مسئولان محترم

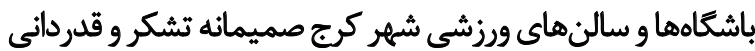

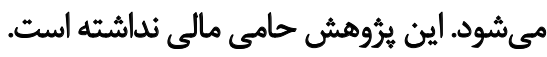




\section{References}

[1] Petersen AMW. The anti-inflammatory effect of exercise. Journal of Applied Physiology. American Physiological Society. 2005; 98(4):1154-62. doi: 10.1152/japplphysiol.00164.2004

[2] Woods JA, Wilund KR, Martin SA, Kistler BM. Exercise, inflammation and aging. Aging and Disease. 2012; 3(1):130-40. PMCID: PMC3320801

[3] Chedraui P, Jaramillo W, Pérez-López FR, Escobar GS, Morocho $\mathrm{N}$, Hidalgo L. Pro-inflammatory cytokine levels in postmenopausal women with the metabolic syndrome. Gynecological Endocrinology. 2010; 27(9):685-91. doi: 10.3109/09513590.2010.521270

[4] Chung HY, Cesari M, Anton S, Marzetti E, Giovannini S, Seo AY, et al. Molecular inflammation: Underpinnings of aging and agerelated diseases. Ageing Research Reviews. 2009(1):18-30. doi: 10.1016/j.arr.2008.07.002

[5] Yasui T, Uemura H, Tomita J, Miyatani Y, Yamada M, Kuwahara A, et al. Association of interleukin-8 with hot flashes in premenopausal, perimenopausal, and postmenopausal women and bilateral oophorectomized women. Journal of Clinical Endocrinology \& Metabolism. 2006; 91(12):4805-8. doi: 10.1210/jc.2006-1100

[6] Cioffi M, Esposito K, Vietri MT, Gazzerro P, D'Auria A, Ardovino I, et al. Cytokine pattern in postmenopause. Maturitas. 2002; 41(3):187-92. doi: 10.1016/s0378-5122(01)00286-9

[7] Michigan A, Johnson TV, Master VA. Review of the relationship between C-reactive protein and exercise. Molecular Diagnosis \& Therapy. 2011(5):265-75. doi: 10.1007/bf03256418

[8] Plaisance EP, Grandjean PW. Physical activity and high-sensitivity C-reactive protein. Sports Medicine. 2006; 36(5):443-58. doi: 10.2165/00007256-200636050-00006.

[9] Nieman DC. Clinical implications of exercise immunology. Journal of Sport and Health Science. 2012; 1(1):12-7. doi: 10.1016/j. jshs.2012.04.004

[10] Mikkelsen UR, Couppé C, Karlsen A, Grosset JF, Schjerling P, Mackey AL, et al. Life-long endurance exercise in humans: Circulating levels of inflammatory markers and leg muscle size. Mechanisms of Ageing and Development. 2013; 134(11-12):531-40. doi: 10.1016/j.mad.2013.11.004

[11] Pereira DS, Mateo ECC, de Queiroz BZ, Assumpção AM, Miranda AS, Felício DC, et al. TNF-a, IL6, and IL10 polymorphisms and the effect of physical exercise on inflammatory parameters and physical performance in elderly women. Age. 2013; 35(6):2455-63. doi: 10.1007/s11357-013-9515-1

[12] Mcfarlin BK, Flynn MG, Campbell WW, Stewart LK, Timmerman KL. TLR4 is lower in resistance-trained older women and related to inflammatory cytokines. Medicine \& Science in Sports \& Exercise. 2004; 36(11):1876-83. doi: 10.1249/01. mss.0000145465.71269.10

[13] Malaguarnera L, Cristaldi E, Vinci M, Malaguarnera M. The role of exercise on the innate immunity of the elderly. European Review of Aging and Physical Activity. 2007; 5(1):43-9. doi: $10.1007 /$ s11556-007-0028-8

[14] Gangemi S, Basile G, Monti D, Merendino RA, Pasquale GD, Bisignano U, et al. Age-related modifications in circulating il-15 levels in humans. Mediators of Inflammation. 2005(4):245-7. doi: 10.1155/mi.2005.245.
[15] Babbitt DM, Diaz KM, Feairheller DL, Sturgeon KM, Perkins AM, Veerabhadrappa P, et al. Endothelial activation microparticles and inflammation status improve with exercise training in African Americans. International Journal of Hypertension. 2013; 2013:1-8. doi: $10.1155 / 2013 / 538017$

[16] Gleeson M, Bishop NC, Stensel DJ, Lindley MR, Mastana SS, Nimmo MA. The anti-inflammatory effects of exercise: mechanisms and implications for the prevention and treatment of disease. Nature Reviews Immunology. 2011; 11(9):607-15. doi: 10.1038/nri3041

[17] Conceição MS, Libardi CA, Nogueira FRD, Bonganha V, Gáspari AF, Chacon-Mikahil MPT, et al. Effects of eccentric exercise on systemic concentrations of pro- and anti-inflammatory cytokines and prostaglandin (E2): comparison between young and postmenopausal women. European Journal of Applied Physiology. 2012; 112(9):3205-13. doi: 10.1007/s00421-011-2292-6

[18] Phillips MD, Patrizi RM, Cheek DJ, Wooten JS, Barbee JJ, Mitchell JB. Resistance training reduces subclinical inflammation in obese, postmenopausal women. Medicine \& Science in Sports \& Exercise. 2012; 44(11):2099-110. doi: 10.1249/ mss.0b013e3182644984

[19] Grabstein K, Eisenman J, Shanebeck K, Rauch C, Srinivasan $\mathrm{S}$, Fung V, et al. Cloning of a T cell growth factor that interacts with the beta chain of the interleukin-2 receptor. Science. 1994; 264(5161):965-8. doi: 10.1126/science.8178155

[20] Quinn LS, Anderson BG. Interleukin-15, IL-15 receptor-alpha, and obesity: concordance of laboratory animal and human genetic studies. Journal of Obesity. 2011:1-8. doi: 10.1155/2011/456347

[21] Lutz CT, Quinn LS. Sarcopenia, obesity, and natural killer cell immune senescence in aging: Altered cytokine levels as a common mechanism. Aging. 2012; 4(8):535-46. doi: 10.18632/aging.100482

[22] Quinn LS, Anderson BG, Strait-Bodey L, Wolden-Hanson T. Serum and muscle interleukin-15 levels decrease in aging mice: Correlation with declines in soluble interleukin- 15 receptor alpha expression. Experimental Gerontology. 2010; 45(2):106-12. doi: 10.1016/j.exger.2009.10.012

[23] Lee S-L. Effect of passive repetitive isokinetic training on Cytokines and hormonal changes. The Chinese Journal of Physiology. 2011; 54(1):55-66. doi: 10.4077/cjp.2011.amm086

[24] Prestes J, Shiguemoto G, Botero JP, Frollini A, Dias R, Leite R, et al. Effects of resistance training on resistin, leptin, cytokines, and muscle force in elderly post-menopausal women. Journal of Sports Sciences. 2009; 27(14):1607-15. doi: 10.1080/02640410903352923

[25] Haghighi A, Hamedinia M. [The comparison of inflammatory markers of related to post menopause in active and non-active postmenopausal women (Persian)]. Olympic. 2011; 18(4):19-28.

[26] Tartibian B, FitzGerald LZ, Azadpour N, Maleki BH. A randomized controlled study examining the effect of exercise on inflammatory cytokine levels in post-menopausal women. Post Reproductive Health. 2015; 21(1):9-15. doi: 10.1177/2053369114565708

[27] Nicklas BJ, Hsu F-C, Brinkley TJ, Church T, Goodpaster BH, Kritchevsky SB, et al. Exercise training and plasma c-reactive protein and interleukin-6 in elderly people. Journal of the American Geriatrics Society. 2008; 56(11):2045-52. doi: 10.1111/j.15325415.2008.01994.x

[28] Hammonds TL, Gathright EC, Goldstein CM, Penn MS, Hughes JW. Effects of exercise on c-reactive protein in healthy pa- 
tients and in patients with heart disease: A meta-analysis. Heart \& Lung: The Journal of Acute and Critical Care. 2016; 45(3):273-82. doi: 10.1016/j.hrtlng.2016.01.009

[29] Xu H, Barnes GT, Yang Q, Tan G, Yang D, Chou CJ, et al. Chronic inflammation in fat plays a crucial role in the development of obesity-related insulin resistance. Journal of Clinical Investigation. 2003; 112(12):1821-30. doi: 10.1172/jci200319451

[30] Fried SK. Omental and subcutaneous adipose tissues of obese subjects release interleukin-6: Depot difference and regulation by glucocorticoid. Journal of Clinical Endocrinology \& Metabolism. 1998; 83(3):847-50. doi: 10.1210/jc.83.3.847

[31] Harkins JM, Moustaid-Moussa N, Chung YJ, Penner KM, Pestka JJ, North CM, et al. Expression of interleukin-6 is greater in preadipocytes than in adipocytes of 3T3-L1 cells and C57BL/6 and OB/OB mice. Journal of Nutrition. 2004; 134(10):2673-7. PMID: 15465765

[32] Church TS, Earnest CP, Thompson AM, Priest EL, Rodarte RQ, Saunders T, et al. Exercise without weight loss does not reduce C-reactive protein. Medicine \& Science in Sports \& Exercise. 2010; 42(4):708-16. doi: 10.1249/mss.0b013e3181c03a43

[33] Okita K. Can exercise training with weight loss lower serum C-reactive protein levels? Arteriosclerosis, Thrombosis, and Vascular Biology. 2004; 24(10):1868-73. doi: 10.1161/01. atv.0000140199.14930.32

[34] Campbell PT, Campbell KL, Wener MH, Wood BL, Potter JD, Mctiernan A, et al.A yearlong exercise intervention decreases CRP among obese postmenopausal women. Medicine \& Science in Sports \& Exercise. 2009; 41(8):1533-9. doi: 10.1249/ mss.0b013e31819c7feb

[35] Della Gatta PA, Garnham AP, Peake JM, Cameron-Smith D. Effect of exercise training on skeletal muscle cytokine expression in the elderly. Brain, Behavior, and Immunity. 2014; 39:80-6. doi: 10.1016/j.bbi.2014.01.006

[36] Heredia JE, Mukundan L, Chen FM, Mueller AA, Deo RC, Locksley RM, et al. Type 2 innate signals stimulate fibro/adipogenic progenitors to facilitate muscle regeneration. Cell. 2013; 153(2):376-88. doi: 10.1016/j.cell.2013.02.053

[37] Bodine SC. Identification of ubiquitin ligases required for skeletal muscle atrophy. Science. 2001; 294(5547):1704-8. doi:10.1126/ science.1065874

[38] Barra NG, Reid S, MacKenzie R, Werstuck G, Trigatti BL, Richards C, et al. Interleukin- 15 contributes to the regulation of murine adipose tissue and human adipocytes. Obesity. 2009; 18(8):1601-7. doi: 10.1038/oby.2009.445

[39] Quinn LS. Interleukin-15: A muscle-derived cytokine regulating fat-to-lean body composition. Journal of Animal Science. 2007; 86(14):75-83. doi: 10.2527/jas.2007-0458

[40] Nielsen AR, Hojman P, Erikstrup C, Fischer CP, Plomgaard P, Mounier R, et al. Association between Interleukin-15 and Obesity: Interleukin-15 as a potential regulator of fat mass. Journal of Clinical Endocrinology \& Metabolism. 2008; 93(11):4486-93. doi: $10.1210 /$ jc.2007-2561

[41] Argiles JM, Lopez-Soriano J, Almendro V, Busquets S, LopezSoriano FJ. Cross-talk between skeletal muscle and adipose tissue: a link with obesity? ChemInform. 2005; 36(11). doi: 10.1002/ chin. 200511290
[42] Jacobi SK, Gabler NK, Ajuwon KM, Davis JE, Spurlock ME. Adipocytes, myofibers, and cytokine biology: New horizons in the regulation of growth and body composition. Journal of Animal Science. 2006; 84(13):E140. doi: 10.2527/2006.8413_supple140x

[43] Carbó N, López-Soriano J, Costelli P, Alvarez B, Busquets S, Baccino FM, et al. Interleukin- 15 mediates reciprocal regulation of adipose and muscle mass: A potential role in body weight control. Biochimica et Biophysica Acta. 2001; 1526(1):17-24. doi: 10.1016/ s0304-4165(00)00188-4

[44] Quinn LS, Anderson BG, Conner JD, Wolden-Hanson T. IL-15 overexpression promotes endurance, oxidative energy metabolism, and muscle PPARס, SIRT1, PGC-1a, and PGC-1 $\beta$ expression in male mice. Endocrinology. 2013; 154(1):232-45. doi: 10.1210/ en.2012-1773

[45] Batista Júnior ML, Lopes RD, Seelaender MCL, Lopes AC. [Anti-inflammatory effect of physical training in heart failure: role of TNF- $\alpha$ and IL-10 (Portuguese)]. Arquivos Brasileiros de Cardiologia. 2009; 93(6):692-700. doi: 10.1590/s0066-782×2009001200021

[46] Ribeiro F, Alves A, Teixeira M, Miranda F, Azevedo C, Duarte $\mathrm{J}$, et al. Exercise training increases interleukin-10 after an acute myocardial infarction: A randomised clinical trial. International Journal of Sports Medicine. 2011;33(3):192-8. doi: 10.1055/s-00311297959

[47] Goldhammer E, Tanchilevitch A, Maor I, Beniamini Y, Rosenschein U, Sagiv M. Exercise training modulates cytokines activity in coronary heart disease patients. International Journal of Cardiology. 2005; 100(1):93-9. doi: 10.1016/j.ijcard.2004.08.073

[48] Oertelt-Prigione S. The influence of sex and gender on the immune response. Autoimmunity Reviews. 2012; 11(6-7):A479A485. doi: 10.1016/j.autrev.2011.11.022

[49] Vural P, Canbaz M, Akgul C. Effects of menopause and postmenopausal tibolone treatment on plasma TNFa, IL-4, IL-10, IL-12 cytokine pattern and some bone turnover markers. Pharmacological Research. 2006; 53(4):367-71. doi: 10.1016/j.phrs.2006.01.005

[50] Yasui T, Maegawa M, Tomita J, Miyatani Y, Yamada M, Uemura $\mathrm{H}$, et al. Changes in serum cytokine concentrations during the menopausal transition. Maturitas. 2007; 56(4):396-403. doi: 10.1016/j.maturitas.2006.11.002

[51] Santos RVT, Viana VAR, Boscolo RA, Marques VG, Santana MG, Lira FS, et al. Moderate exercise training modulates cytokine profile and sleep in elderly people. Cytokine. 2012; 60(3):731-5. doi: 10.1016/j.cyto.2012.07.028

[52] Arai MH, Duarte AJ, Natale VM. The effects of long-term endurance training on the immune and endocrine systems of elderly men: The role of cytokines and anabolic hormones. Immunity \& Ageing. 2006; 3:9. doi: 10.1186/1742-4933-3-9

[53] Vieira VJ, Hu L, Valentine RJ, McAuley E, Evans EM, Baynard $\mathrm{T}$, et al. Reduction in trunk fat predicts cardiovascular exercise training-related reductions in C-reactive protein. Brain, Behavior, and Immunity. 2009; 23(4):485-91. doi: 10.1016/j.bbi.2009.01.011

[54] Colbert LH, Visser M, Simonsick EM, Tracy RP, Newman AB Kritchevsky SB, et al. Physical Activity, exercise, and inflammatory markers in older adults: Findings from The health, aging and body composition study. Journal of the American Geriatrics Society. 2004; 52(7):1098-104. doi: 10.1111/j.1532-5415.2004.52307.x

[55] Nieman DC, Pedersen BK. Nutrition and exercise immunology. Florida: CRC Press; 2000. 
